\title{
Aneurysms in the ciliary body band confirmed by fluorescein gonioangiography
}

\author{
RYOZO KIMURA, YOICHI TAKAKU, AND KUMIKO SUZUKI \\ From the Department of Ophthalmology, School of Medicine, Tohoku University, 1-1 Seiryo-cho, Sendai 980, \\ Japan
}

SUMMARY Fluorescein gonioangiography was performed in a protracted case of Vogt-KoyanagiHarada syndrome. Small, discrete, reddish lesions in the ciliary body band were confirmed to be aneurysms because they showed sharply defined hyperfluorescence shortly after fluorescein injection.

One of the authors (R.K.) has described, in patients with granulomatous uveitis, ${ }^{1}$ whitish lesions in the ciliary body band which showed hyperfluorescence shortly after fluorescein injection. The hyperfluorescent dots were considered to be granulomas in the ciliary body band. The purpose of this communication is to describe small, discrete, reddish lesions which have shown sharply defined hyperfluorescence shortly after fluorescein injection in a protracted case of VogtKoyanagi-Harada syndrome.

\section{Case report}

Complaining of blurred vision in both eyes, a 27 year-old man was seen by an ophthalmologist 6 years earlier. With the diagnosis of Vogt-Koyanagi-Harada syndrome systemic corticosteroids were instituted. A week later he was referred to our clinic. When he was seen in our clinic, vision was 20/40 with myopic correction of $-6.5 \mathrm{D}$ in both eyes. Applanation pressure was 15 in the right and $13 \mathrm{mmHg}$ in the left eye. The cornea was clear except for some keratic precipitates in both eyes. The anterior chamber was deep in both eyes. Biomicroscopy revealed flare and cells in both anterior chambers. Ophthalmoscopy revealed multiple serous retinal detachments in both eyes. Results of laboratory examinations, including complete blood cell count, electrolytes, cholesterol, glucose, and liver and renal functions, were normal. A serological syphilitic test, the rheumatoid arthritis titre, and Creactive protein value were negative. An antistreptolysin 0 value of 50 Todd units was also obtained. Although he had complained of buzzing in the ears, otological examinations revealed no abnormal findCorrespondence to Dr R. Kimura. ings. The diagnosis of Vogt-Koyanagi-Harada syndrome was made. Systemic corticosteroids were given over the following 2 months, resulting in subsidence of the disease.

Since then the patient was seen on follow-up examinations by an ophthalmologist to have suffered several attacks of mild iritis. He was referred to our clinic again on 26 May 1981 because of increased intraocular pressure in the left eye. On examination vision was $20 / 25$ with myopic correction of $-9 \mathrm{D}$ in the right and $20 / 40$ with myopic correction of $-8 \mathrm{D}$ in the left eye. Applanation pressure was 12 in the right and $55 \mathrm{mmHg}$ in the left eye. The anterior chamber was deep in both eyes. The cornea was clear in the right and slightly oedematous in the left eye. Biomicroscopy after peroral $50 \%$ glycerol $(2 \mathrm{ml} / \mathrm{kg})$ revealed clear aqueous humour in both eyes. The lens in both eyes showed slight posterior subcapsular opacities, probably due to prolonged use of corticosteroids. Ophthalmoscopy revealed in both eyes a diffusely red fundus associated with numerous depigmented foci. Fluorescein angiography of the fundus showed no newly formed retinal or choroidal vessels nor leakage of dye. Gonioscopy revealed a wide open angle with wide ciliary body band in both eyes. However, newly formed angle vessels were seen in both eyes extending from the angle recess toward the trabecular meshwork, associated with small discrete reddish lesions in the ciliary body band. The left eye was affected the more severely. Fluorescein gonioangiography showed clearly the newly formed angle vessels shortly after fluorescein injection in both eyes (Figs. 1 and 2). The reddish lesions in the ciliary body band also showed a sharply defined hyperfluorescence shortly after fluorescein injection in both eyes (Figs. 1 and 2). Both 
Fig. 1 Fluorescein gonioangiogram in the lower nasal quadrant of the right eye 15 seconds afterfluorescein injection, showing hyperfluorescent dots (small white arrows) corresponding to the reddish lesions associated with newly formed vessels. Large white arrows indicate the iris root, and black arrows indicate Schwalbe's line.

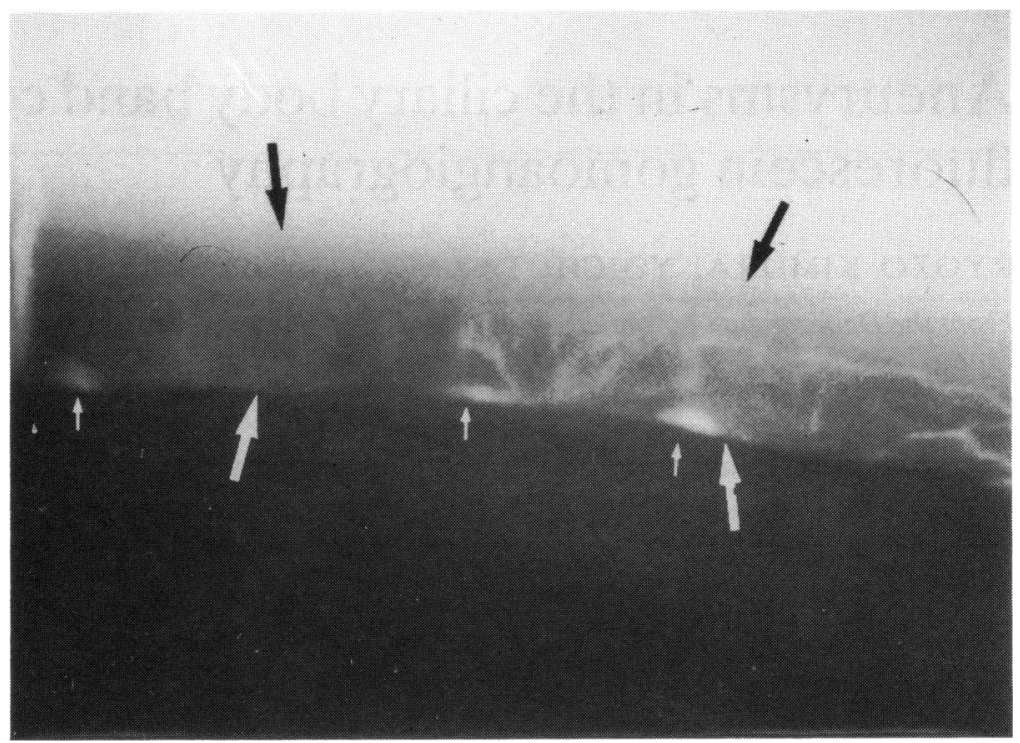

visual fields were normal. Oral acetazolamide $250 \mathrm{mg}$ once in the morning was instituted, resulting in return to normal of the intraocular pressure in the left eye. Since then examinations of the patient at intervals of a month have revealed no changes in either eye.

\section{Discussion}

In previous papers ${ }^{12}$ one of the authors (R.K.) presented several characteristic findings obtained by the original method of fluorescein gonioangiography ${ }^{2}$ as follows: (i) no staining or leakage in normal persons; (ii) increased visibility of newly formed angle vessels; (iii) leakage from the recessed angle in cases with traumatic angle recession; (iv) vigorous leakage from the angle recess in a case with Behçet's disease during an attack of uveitis; (v) hyperfluorescent dots in the ciliary body band corresponding to the whitish lesions in cases with granulomatous uveitis. Thus fluorescein gonioangiography as well as fundus angiography offers useful informations in the diagnosis of eye disorders. In the present study we have dealt with reddish lesions
Fig. 2 Fluorescein gonioangiogram in the lower temporal quadrant of the left eye 15 seconds after fluorescein injection, showing hyperfluorescent dots (small white arrows) corresponding to the reddish lesions associated with newly formed vessels. Large white arrows indicate the iris root, and black arrows indicate Schwalbe's line.

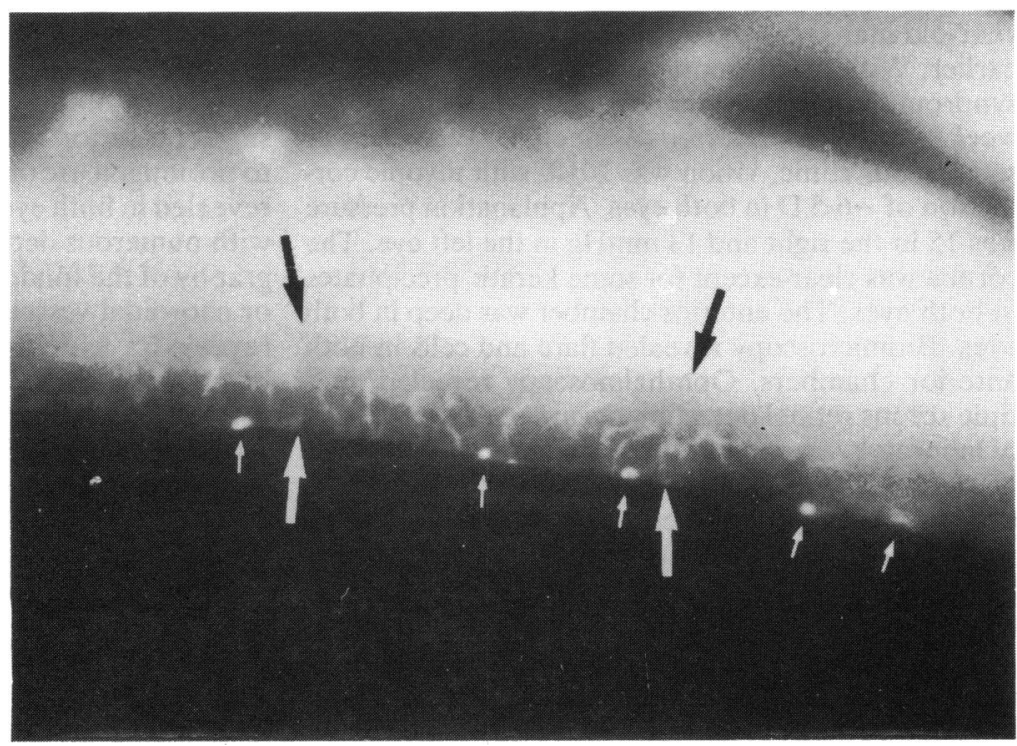


in the ciliary body band. There are several reports of haemorrhages in the angle. ${ }^{34}$ For example, Moreu (cited by Troncoso ${ }^{3}$ ) described cases of syphilitic iritis with intense congestion of the root of the iris, ciliary body oedema, and small haemorrhages. To the best of our knowledge, however, there are no reports of aneurysms in the angle. Their absence may be attributed to a lack of gonioangiographic studies, because it is well known that only fluorescein angiography can differentiate aneurysms from haemorrhages clinically. In the present study aneurysms in the ciliary body band associated with newly formed vessels were demonstrated by means of fluorescein gonioangiography.

\section{References}

1 Kimura R. Hyperfluorescent dots in the ciliary body band in patients with granulomatous uveitis. Br J Ophthalmol 1982: 66: 322-5.

2 Kimura R. Fluorescein goniophotography. Glaucoma 1980; 2: 359-67.

3 Troncoso MU. A treatise on gonioscopy. Philadelphia: Davis. 1948: 164-5.

4 Chandler PA. Grant WM. Glaucoma. 2nd ed. Philadelphia: Lea and Febiger, 1979; 45: 54-5. 